\title{
Can Mirror-Reading Reverse the Flow of Time?
}

\author{
Daniel Casasanto ${ }^{1,2}$ and Roberto Bottini ${ }^{1,3}$ \\ ${ }^{1}$ Max Planck Institute for Psycholinguistics, Neurobiology of Language Group, \\ Nijmegen, NL \\ ${ }^{2}$ Donders Center for Brain, Cognition, and Behavior, Radboud University, Nijmegen, NL \\ ${ }^{3}$ University of Bergamo, Department of Human Sciences, Bergamo, IT \\ daniel.casasanto@mpi.nl, \\ roberto.bottini@unibg.it
}

\begin{abstract}
Across cultures, people conceptualize time as if it flows along a horizontal timeline, but the direction of this implicit timeline is culture-specific: in cultures with left-to-right orthography (e.g., English-speaking cultures) time appears to flow rightward, but in cultures with right-to-left orthography (e.g., Arabic-speaking cultures) time flows leftward. Can orthography influence implicit time representations independent of other cultural and linguistic factors? Native Dutch speakers performed a space-time congruity task with the instructions and stimuli written in either standard Dutch or mirror-reversed Dutch. Participants in the Standard Dutch condition were fastest to judge past-oriented phrases by pressing the left button and future-oriented phrases by pressing the right button. Participants in the Mirror-Reversed Dutch condition showed the opposite pattern of reaction times, consistent with results found previously in native Arabic and Hebrew speakers. These results demonstrate a causal role for writing direction in shaping implicit mental representations of time.
\end{abstract}

Keywords: Culture, Metaphor, Orthography, Space, Time.

\section{Introduction}

Space and time are intertwined in the human mind, as they are in the physical world. The theory that people use spatial representations to think about time, first inspired by patterns in metaphorical language $[1,2]$, is now supported by numerous behavioral and neuroscientific experiments [e.g., 3, 4, 5, 6, 7].

Yet, the way people use space to talk about time is not necessarily the same way they use space to think about it. In English and many other languages, metaphors suggest that time flows along the sagittal (front-back) axis: deadlines lie ahead of us or behind us; we can look forward to our golden years or look back on our salad days. Other languages also make use of the vertical axis to talk about time. In Mandarin Chinese, 'the up month' means a month earlier and 'the down month' a month later [8]. Yet, no known spoken language uses the lateral (left-right) axis to talk about time conventionally, and invented left-right metaphors for time sound nonsensical: Monday comes before Tuesday, not to the left of Tuesday [9].

Despite the total absence of left-right metaphors in spoken language, there is strong evidence that people implicitly associate time with left-right space. Furthermore, the

C. Hölscher et al. (Eds.): Spatial Cognition VII, LNAI 6222, pp. 335-345, 2010.

(C) Springer-Verlag Berlin Heidelberg 2010 
direction in which time flows along people's imaginary timeline varies systematically across cultures. In one study, Tversky, Kugelmass, \& Winter [10] asked children and adults to place stickers on a page to indicate where breakfast and dinner should appear relative to the lunch sticker, in the middle of the page. Whereas English speakers placed breakfast on the left and dinner on the right of lunch, Arabic speakers preferred the opposite arrangement. Fuhrman and Boroditsky [11] showed a similar pattern in a reaction time (RT) task. English- and Hebrew-speaking participants judged whether the second of two pictures showed an earlier or later stage of an unfolding event. English speakers' judgments were fastest when earlier was mapped to the left button and later to the right, but Hebrew speakers showed the opposite pattern. Ouellet, et al. [12] asked Spanish and Hebrew speakers to judge auditorily presented words referring to the past or future with either their left or right hand, and found a similar reversal of the lateral space-time mapping across groups.

These experimental data reflect patterns that can be found in spontaneous behavior, as well. When English speakers produce co-speech gestures they tend to use the lateral axis for time, much more often than the sagittal axis $[13$, see also $14 ; 9 ; 15]$. Earlier times are on the left and later times on the right of body-centered space. Preliminary data from our lab suggests that Spanish speakers' gestures follow a similar pattern, but Arabic speakers' spontaneous gestures show the reverse mapping (Romàn, Casasanto, Jasmin, \& Santiago, in prep).

Across cultures, the direction in which time flows along the mental timeline varies predictably with the orthography of the dominant language: time flows rightward in cultures whose literate members use a left-to-right orthography and leftward in cultures that use a right-to-left orthography. Yet, despite this clear correlation, it is not known to what extent reading and writing direction is a cause or an effect of crosscultural variation in implicit space-time mappings.

In principle, a culture's writing system could emerge with one directionality or another as a consequence of culture-specific conceptions of time -- not the other way around. This seems especially plausible for cultures where literacy (or mass-literacy) is a recent development. Alternatively, directionality in both orthography and in thought could arise due to cultural bootstrapping from material artifacts like calendars (whether the calendar is a grid on a piece of paper, knots on a string, notches on a branch, etc.) or other devices for keeping track of time (e.g., a solar clock) or number (e.g., a horizontal abacus) [16]. Cultural practices tend to covary: groups who write from left to right often spatialize time on calendars and numbers on graphs from left to right, as well. Based on correlational data, it is not possible to determine whether experience reading or writing plays any causal role in fixing the direction of implicit space-time mappings.

Here we performed an experimental intervention to determine whether experience with reading a left-to-right or right-to-left orthography is sufficient to determine the direction of people's implicit associations from space to time. Native Dutch speakers were assigned to perform one of two space-time congruity tasks. In one task (Experiment 1), participants saw past-oriented phrases (e.g. a year earlier) and futureoriented phrases (e.g. a decade later) appear on the screen one at a time, in standard Dutch orthography. As soon as each phrase appeared, they pressed a button (located on the left or right of a keyboard) to indicate the temporal reference of the phrase (past or future). Each participant performed two blocks: in one block the left-right key 
mapping required responses that were congruent with a left-to-right flow of time, and in the other responses were congruent with a right-to-left mapping. The order of blocks was counterbalanced across participants. We predicted that, on average, participants would show an RT advantage for responses consistent with standard Dutch orthography (left-to-right).

The other task (Experiment 2) was identical to the first, with one exception: all instructions and stimuli were presented in mirror-reversed text. Reading requires scanning the page in a particular direction: normally for Dutch speakers reading each line of a text requires moving the eyes gradually from the left to the right side of the page or the computer screen. As such, moving rightward in space is tightly coupled with 'moving' later in time. We reasoned that if the habit of reading from left-to-right contributes to an implicit left-to-right mapping of time in readers' minds, then practice reading in the opposite direction should weaken and eventually reverse this mapping.

\section{Space-Time Congruity with Standard Orthography}

In Experiment 1, all instructions and stimuli were presented in standard Dutch orthography. We conducted Experiment 1 to validate the use of this space-time congruity paradigm in native Dutch speakers, and to provide a comparison group for the mirrorreading group.

\subsection{Methods}

Participants. Native Dutch speakers $(\mathrm{N}=32)$ performed Experiment 1 in exchange for payment.

Stimuli. Temporal phrases were constructed in Dutch, each with 3 words. The first word was an indefinite article, the second word a temporal interval, (tr., second, moment, minute, hour, day, week, month, season, year, decade, century, millennium), and the third word a temporal modifier (tr., before, after, earlier, later). The twelve temporal intervals were fully crossed with the four temporal modifiers to produce 48 temporal phrases (e.g., a day before; a century after; a year earlier; a week later). Half of the phrases referred to an earlier (past-oriented) interval of time (i.e., if the modifier was earlier or before), and the other half referred to a later (future-oriented) interval (i.e., if the modifier was later or after). Two of the modifiers were spatial terms used metaphorically (before, after), and the other two were purely temporal terms with similar meanings (earlier, later). Phrases were presented in the center of a Macintosh laptop screen (resolution=1024x768), in black 48-point Arial font, on a white background.

Apparatus. Participants were seated at a desk. Two A4 Xerox paper boxes were stacked on the desk, and a laptop computer was secured on top of them, to raise the screen to approximately the participants' eye-level. A standard USB keyboard was mounted horizontally on the side of the upper box, with the keys facing the participant, at about shoulder level. The keyboard was covered with a sheet of black plastic with holes that exposed only the three keys needed for responses: the "A" key on the left, the "apostrophe" key on the right, and the " $\mathrm{H}$ " hey in the middle. The middle key was aligned with the center of the laptop screen, and the left and right keys were 
equidistant from it. The left key was covered with a blue sticker and the right key a red sticker, or vice versa, with the key colors counterbalanced across subjects.

Procedure. The experiment consisted of two blocks. In each block, each of the 48 temporal phrases was presented once, for a total of 96 trials. Written instructions appeared on the screen before each block. In one of the blocks, participants were instructed that as soon as each phrase appeared, they should press the blue button if the phrase referred to an interval of time in the past (e.g., a week earlier) and the red button if it referred to an interval of time in the future (e.g., a week later). In the other block, the mapping between the red/blue keys and pastward/futureward phrases was reversed. To ensure that participants remembered the correct color-time mapping, after reading the instructions they were required to rehearse the correct color-time mapping aloud 5 times, before each block (e.g., "past=blue, past=blue, past=blue, etc.; future $=$ red, future $=$ red, future $=$ red, etc.)

At the beginning of each trial the word 'ready' appeared in the center of the screen and remained there until the participant pressed the middle white button. 'Ready' was then replaced by a fixation cross. Participants were instructed to hold down the white button for as long as the fixation was shown. Its duration was varied randomly from $300-450 \mathrm{~ms}$, in $50 \mathrm{~ms}$ increments, to make its duration unpredictable and discourage anticipatory movements. The fixation was then replaced by one of the 48 temporal phrases. Participants were instructed to press the colored button corresponding to the temporal reference of the phrase as quickly and accurately as possible. The phrase remained on the screen until the participant responded, at which time it was replaced by the 'ready' message to begin the next trial.

Participants pressed buttons with the index finger of the dominant (writing) hand. To ensure they would used the same hand for both rightward and leftward responses, participants were required to sit on their non-dominant hand.

The spatial direction of responses was never mentioned, but one colored button was on the right and the other on the left of the middle white button. Therefore, in one block pressing the correctly colored button called for a movement that was congruent with the space-time mapping encoded in standard Dutch orthography (e.g., pressing the blue button for a pastward phrase when the blue button was on the left); in the other block pressing the correctly colored button called for an incongruent movement (e.g., pressing the blue button for a futureward phrase when the blue button was on the left). The order of congruent-movement and incongruent-movement blocks was counterbalanced across participants. The space-time congruity effect was computed for each subject by comparing response times during Congruent and Incongruent responses (between-blocks, within-items). Testing lasted about 10 minutes.

\subsection{Results and Discussion}

Participants pressed the correct button on $96 \%$ of trials. Only accurate responses were analyzed. This resulted in the removal of $4 \%$ of the data. Responses greater than 5000 ms were also excluded, which resulted in the removal of $0.2 \%$ of the accurate trials.

A 2 X 2 ANOVA was conducted with Congruity of Movement Direction (Congruent with Time flowing leftward, Congruent with time flowing rightward) and Block (Block 1, Block 2) as within-subject and within-item factors. There was a highly significant main effect of Congruity $\left(F_{1}(1,15)=30.56, p=.0001 ; F_{2}(1,47)=119.38, p=.0001\right)$. There 
was no main effect of Block $\left(F_{1}(1,15)=0.75, n s ; F_{2}(1,47)=2.99, n s\right)$. The Congruity X Block interaction was significant by items but not by subjects $\left(F_{1}(1,15)=1.41, n s\right.$; $\left.F_{2}(1,47)=6.27, p=.02\right)$.

Congruity of Movement was then compared within each block (Block 1: $F_{1}(1,30)=9.62, p=.004 ; F_{2}(1,47)=116.31, p=.0001$; Block 2: $F_{1}(1,30)=3.64, p=.07$; $\left.F_{2}(1,47)=32.55, p=.0001\right)$. Mean RTs are shown in figure 1 .

Overall, there was a strong effect of Congruity. Participants responded faster when the mapping between the color of the buttons and the temporal reference of the phrases required leftward movements for past-oriented phrases and rightward movements future-oriented phrases. This space-time congruity effect is similar to effects found previously in English and Spanish speakers (e.g., Torralbo, et al., 2006; Weger \& Pratt, 2008). We are not aware of previous studies showing this effect in Dutch speakers, but given the correlation between writing direction and the direction of the space-time mappings across cultures, we had no reason to expect that Dutch speakers should perform differently from speakers of other languages that use a Roman alphabet.

For our present purposes, it is important that this paradigm produced a congruity effect in the same direction for both blocks. Having shown that this task provides clear evidence for the implicit space-time mapping typically found in left-to-right reading cultures, we can proceed to test effects of exposure to an orthography in which 'progress' along a spatio-temporal continuum proceeds in the opposite direction.

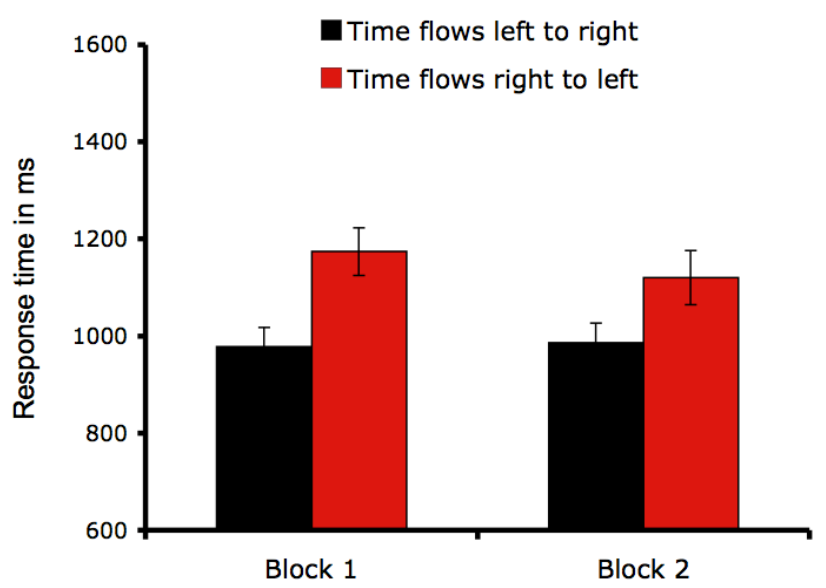

Fig. 1. Results of Experiment 1. When reading standard orthography, participants were faster to judge the temporal reference of phrases when the left-right button press responses were consistent with the rightward flow of time along an implicit mental timeline (left=earlier, right=later). Error bars indicate s.e.m.

\section{Space-Time Congruity with Mirror-Reversed Orthography}

To test for a causal role of orthography in the mental representation of temporal order, we replicated Experiment 1 in a new group of participants using stimuli and instructions presented in mirror-reversed font. 


\subsection{Methods}

Participants. A new sample of native Dutch speakers $(\mathrm{N}=32)$ performed Experiment 2 in exchange for payment.

Materials and Procedure. Materials and procedures were identical to Experiment 1, with one exception. All instructions and stimuli were presented mirror-reversed. Testing lasted about 15 minutes.

\subsection{Results and Discussion}

Participants pressed the correct button on $97 \%$ of trials. Only accurate responses were analyzed. This resulted in the removal of 3\% of the data. Responses greater than 5000 ms were also excluded, which resulted in the removal of $4 \%$ of the accurate trials trials.

A 2 X 2 ANOVA was conducted with Congruity of Movement Direction (Congruent with Time flowing leftward, Congruent with time flowing rightward) and Block (Block 1, Block 2) as within-subject and within-item factors. There was no main effect of Congruity $\left(F_{1}(1,15)=.79, n s ; F_{2}(1,47)=2.29, n s\right)$. There was a highly significant effect of Block $\left(F_{1}(1,15)=66.37, p=.0001 ; F_{2}(1,47)=321.81, p=.0001\right)$, and crucially, a highly significant Congruity $X$ Block interaction $\left(F_{1}(1,15)=31.89\right.$, $\left.p=.0001 ; F_{2}(1,47)=206.56, p=.0001\right)$.

Congruity of Movement was then compared within each block (Block 1: $F_{1}(1,30)=5.00, p=.03 ; F_{2}(1,47)=98.36, p=.0001 ;$ Block $2: F_{1}(1,30)=3.02, p .=.09$; $\left.F_{2}(1,47)=125.21, p=.0001\right)$. Mean RTs are shown in figure 2 .

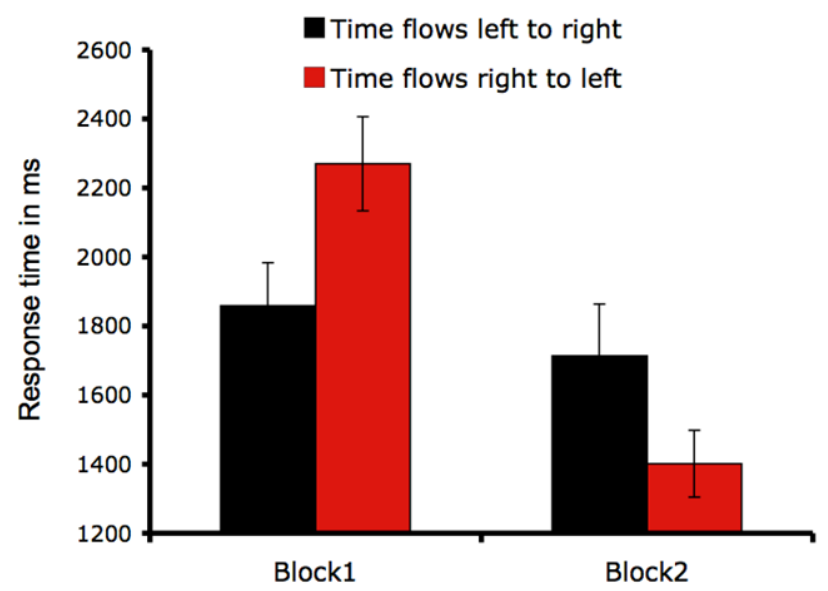

Fig. 2. Results of Experiment 2. In the first block, the space-time congruity effect was similar to that observed in Experiment 1. But by the second block of reading mirror-reversed orthography, this congruity effect reversed. Participants were faster to judge the temporal reference of phrases when the left-right button press responses were consistent with a leftward flow of time along an implicit mental timeline (left=later, right=earlier). Error bars indicate s.e.m. 
Finally, we compared the congruity effects found in Experiment 1 and Experiment 2 using a 2 X 2 X 2 ANOVA with Congruity and Block as within-subject/within-item factors and Orthography (Standard orthography, Mirror-reversed orthography) as a within-subject/within-item factor. Consistent with the prediction that orthography can influence mental representations of time, we find a highly significant 3-way interaction $\left(F_{1}(1,30)=22.71, p=.0001 ; F_{2}(1,94)=125.38, p=.0001\right)$. By subtracting the RTs during trials where movements were congruent with the leftward flow of time from RTs during trials where movements were congruent with the rightward flow of time (RT_rightward - RT_leftward), this 3-way interaction can be simplified, and conceptualized as a 2-way interaction of Block X Orthography (see figure 3).

As is evident from figure 3, the absolute values (ABS) of the congruity effects in both blocks of Experiment 2 are greater than the ABS of the effects in Experiment 1. This was not expected, and although it is not relevant to our experimental hypothesis, it bears further investigation. On one possible explanation, congruity effects may result from a failure of cognitive control; that is, they result from participants' inability to ignore the irrelevant spatial dimension of their responses when judging the temporal reference of the stimuli. The cross-dimensional effect of space on time judgments may have been greater in Experiment 2 because cognitive control resources were taxed by reading backwards, contrary to habit.

Although the dominant space-time mapping in Dutch culture continued to influence RTs during the first block of Experiment 2, by the second block exposure to mirror-reversed writing was sufficient to reverse the congruity effect. Since this is the first experiment to test for a causal influence of writing direction on time representation, we did not have any a priori prediction about how much experience with

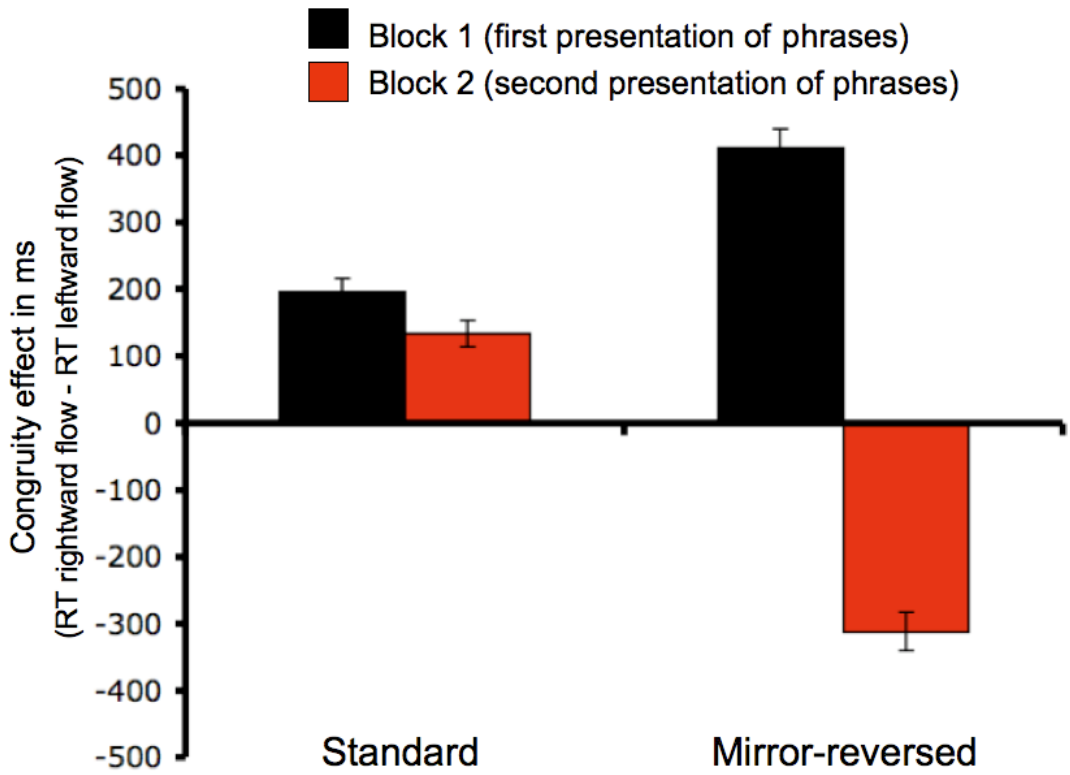

Fig. 3. Congruity effects (RT_rightward - RT_leftward) across blocks for Experiment 1 (left) and Experiment 2 (right). Error bars indicate s.e.m. 
reversed orthography would be needed to produce a significant change in the congruity effect, nor could we predict whether the congruity effect would be reversed or merely diminished. To support our hypothesis, it would have been sufficient to show a reduction in the left-to-right congruity effect from Block 1 to Block 2 that was greater in Experiment 2 than in Experiment 1. However, the fact that the congruity effect completely reversed here provides a particularly clear demonstration that even brief experience with one orthography or another can influence people's implicit spatial representations of time. [For compatible evidence of the flexibility of spacetime metaphors in language and thought, see $4,8,17,1 ; 18,10]$.

\section{General Discussion}

It is now well established that people activate implicit associations between space and time when processing temporal language, and that the specifics of these associations vary systematically across cultures $[11,12,10]$. Since time is not associated with leftright space in any known linguistic metaphors, it is unlikely that these culture-specific mappings are learned through experience with spoken language. ${ }^{1}$ Here we tested whether orthography can play a causal role in fixing the direction in which time flows along the imaginary mental timeline. Experiment 1 showed that, when exposed to temporal phrases presented in standard left-to right orthography, Dutch speakers implicitly associated earlier time intervals with leftward movements and later time intervals with rightward movements, consistent with previous findings in members of other cultures that use the Roman writing system.

However, when exposed to several minutes of mirror-reversed writing, Dutch participants began to show space-time congruity effects that revealed a reversal of their normally dominant implicit space-time mapping. By the second time they were judging each of the 48 temporal phrases (Block 2 of Experiment 2), participants were faster to make responses when key presses associated earlier events with rightward movements and later events with leftward movements -- a pattern observed previously in speakers of Hebrew, which is written from right to left. It appears that experience reading a right-to-left orthography (which requires the reader to 'progress' leftward across the screen with his/her eyes) is sufficient to reverse the flow of time in the reader's mind, at least transiently.

Although this rapid retraining of a space-time association stored in long-term memory may seem surprising, it is not unprecedented. In one study, Boroditsky [8] found that horizontal spatial primes facilitated English speakers' judgments of temporal sentences (e.g., April comes earlier than May) more than vertical primes did, but found the opposite pattern in Mandarin speakers, consistent with the difference between these languages in the prevalence of horizontal and vertical metaphors for time. To test whether linguistic experience could affect these mappings, she trained a new group of English speakers to use Mandarin-like vertical spatial metaphors for time. After brief training, English speakers showed a pattern of priming similar to native Mandarin speakers.

${ }^{1}$ Although spoken languages do not use the lateral axis for time, some signed languages do [23]. 
In a test of a different set of space-time metaphors Casasanto [17] and colleagues showed that when English and Greek speakers perform non-linguistic duration reproduction tasks, they show language-specific patterns of cross-dimensional interference from space. Whereas English speakers have a harder time screening out interference from (1-dimensional) spatial distance, Greek speakers have more difficulty screening out interference of (3-dimensional) volume. This pattern was predicted based on the relative prevalence and productivity of distance and volume metaphors for duration across languages (e.g., a long time (like a long rope); a large amount of time (like $a$ large amount of water)). To find out whether using volume metaphors could cause the volume-interference found in Greeks, US English speakers were trained to use Greeklike volume metaphors for time. Results showed that after one brief (but concentrated) training session, English participants showed a pattern of cross-dimensional interference from volume in a low-level psychophysical task that was statistically indistinguishable from the pattern seen in native Greek speakers.

Time is not the only domain that appears to be mentally represented, in part, through spatial metaphors (which may or may not correspond to linguistic metaphors). Emotional valence is also spatialized on a left-right axis: whereas righthanders tend to associate the right hand and the right side of space with positive things and the left with bad, left-handers show the opposite set of implicit associations [19]. It was proposed that this mapping arises due to asymmetries in motor fluency: people like things on their dominant side better because they can interact with things on that side more easily. To test this proposal, Casasanto [20] asked right-handers to perform a 2-part training task. In the first part, they arranged dominoes according to a symmetrical pattern on a tabletop, standing them on end, moving both hands in synchrony. The challenge was that they were randomly assigned to wear a bulky ski glove one hand or the other while performing the task, which either enhanced their natural right-handedness or made them temporarily more skillful with their left hand.

After 12 minutes of this asymmetric motor experience, participants were taken to a different room by a different experimenter for some ostensibly unrelated questionnaire studies, one of which tested implicit associations between space and valence. This questionnaire was shown previously to produce distinctive patterns of judgments in rightand left-handers [19]. Participants whose training experience preserved their natural dominance showed the typical right-handers' pattern. But participants who had worn the skiglove on their right hand during training, becoming transiently left-handed, produced a pattern of responses that was indistinguishable from natural lefties'.

We are aware of one training study that manipulated writing direction in order to test a role for orthography in the spatial representation of gender and agency. Several studies suggest that males (seen as more agentive) tend to be represented to the left of females in the minds of people who speak left-to-right languages like English, but not for speakers of right-to-left languages like Arabic [21]. Yet, Suitner [21] showed that this spatial bias can be nullified in speakers of Italian who are trained to perform a leftward writing exercise, reversing not only their habitual writing direction but also their habitual associations of gender, agency, and space.

How enduring are these training effects? Presumably, without further reinforcement of the new habits, participants who show rapid training effects will also revert to their long-term habits rapidly. Exactly how soon remains a question for further research. Depending on the goal of the training manipulation, the durability of the behavioral 
change may matter more or less. In the present study the goal was to test the sufficiency of a proposed cause of cross-cultural differences. The total reversal of the congruity effect as a function of reading experience demonstrates that orthography can, indeed, influence the implicit spatial representation of time. This simple demonstration that orthography can play a causal role in directing the flow of time along the mental timeline would serve its theoretical goal even if the effect were quickly reversed when participants resumed normal reading habits.

How best to characterize the learning mechanisms that afford this representational plasticity remains another open question. It may be fruitful to consider the changes participants undergo in Experiment 2 in terms of a hierarchical Bayesian model [22]. To sketch this suggestion briefly, people's associations between space and time could be characterized as intuitive hypotheses. Based on ordinary reading experience, Dutch speakers form the hypothesis that by default events unfold from left to right. Yet after training, they appear to entertain the hypothesis that events unfold from right to left.

To explain how participants can switch from one hypothesis to a contradictory hypothesis (and presumably switch back) so quickly, it may help to posit that they also entertain a more enduring overhypothesis, of which both the 'Dutch-like' and 'Arabic-like' space-time associations are specific instances. The overhypothesis could be that time is associated with motion along a linear path. Such a belief would be well supported by observable correlations in the physical world: spatial succession is a reliable index of temporal succession.

Consistent with this proposal, we suggest that if orthography is responsible for determining the direction in which time flows along people's left-right mental timelines, this directional mapping likely builds upon a prior less-specific space-time association, which arises (either in developmental or evolutionary time) from space-time correlations that have no particular directionality: on any trajectory, it is the case that as a moving object travels farther, more time passes. The hierarchical model can potentially help to explain how 'mental metaphors' linking space-time can be universal at one level of level of description but culture-specific at another.

Acknowledgments. This research was supported in part by the Max Planck Gesellschaft and by an NRSA fellowship (\#F32MH072502) and by a grant from the Spanish Ministry of Education and Science (\#SEJ2006-04732/PSIC, DGI) to DC.

\section{References}

1. Clark, H.H.: Space, time, semantics and the child. In: Moore, T.E. (ed.) Cognitive Development and the Acquisition of Language, pp. 27-63. Academic Press, New York (1973)

2. Lakoff, G., Johnson, M.: Metaphors We Live by. The Chicago University Press, Chicago (1980)

3. Basso, G., Nichelli, P., Frassinetti, F., di Pellegrino, G.: Time perception in a neglected space. Neuroreport 7, 2111-2114 (1996)

4. Boroditsky, L.: Metaphoric structuring: understanding time through spatial metaphors. Cognition 75(1), 1-28 (2000)

5. Casasanto, D., Boroditsky, L.: Time in the Mind: Using space to think about time. Cognition 106, 579-593 (2008) 
6. Torralbo, A., Santiago, J., Lupiáñez, J.: Flexible conceptual projection of time onto spatial frames of reference. Cog. Sci. 30, 749-757 (2006)

7. Weger, U.W., Pratt, J.: Time flies like an arrow: Space-time compatibility effects suggest the use of a mental time-line. Psychonomic Bulletin \& Review 15, 426-430 (2008)

8. Boroditsky, L.: Does language shape thought? English and Mandarin speakers' conceptions of time. Cog. Psych. 43(1), 1-22 (2001)

9. Cienki, A.: Metaphoric gestures and some of their relations to verbal metaphoric expressions. In: Koening, J.-P. (ed.) Discourse and cognition: Bridging the gap, pp. 189-204. CSLI Publications, Stanford (1998)

10. Tversky, B., Kugelmass, S., Winter, A.: Cross-cultural and developmental trends in graphic productions. Cog. Psych. 23, 515-557 (1991)

11. Fuhrman, O., Boroditsky, L.: Mental time-lines follow writing direction: Comparing English and Hebrew speakers. In: Proceedings of 29th Annual Conference of the Cognitive Science Society, Nashville, TN (2007)

12. Ouellet, M., Santiago, J., Israeli, Z., Gabay, S.: Is the future the right time? Exp. Psych. (in press)

13. Casasanto, D.: When is a linguistic metaphor a conceptual metaphor? In: Evans, V., Pourcel, S. (eds.) New Directions in Cognitive Linguistics, pp. 127-145. John Benjamins, Amsterdam (2009)

14. Boroditsky, L.: Do English and Mandarin Speakers Think Differently About Time? In: Proceedings of the 31th Annual Conference of the Cognitive Science Society, pp. 575580. Lawrence Erlbaum Associates, Hillsdale (2008)

15. Cooperrider, K., Nunez, R.: Across time, across the body Transversal temporal gestures. Gesture 9, 181-206 (2009)

16. Dehaene, S.: The number sense. Oxford University Press, New York (1999)

17. Casasanto, D.: Who's afraid of the Big Bad Whorf? Cross-linguistic differences in temporal language and thought. Language Learning 58(1), 63-79 (2008)

18. Evans, V.: The structure of time: Language, meaning and temporal cognition. John Benjamins, Amsterdam (2004)

19. Casasanto, D.: Embodiment of Abstract Concepts: Good and bad in right- and left-handers. JEP: General 138(3), 351-367 (2009)

20. Casasanto, D.: Motor experience shapes abstract concepts. In: Proceedings of the 50th Annual Meeting of the Psychonomic Society, Boston, MA (2009)

21. Suitner, C.: Where to place social targets? Stereotyping and Spatial Agency Bias. Doctoral dissertation, Department of Psychology, University of Padova (2009)

22. Kemp, C., Perfors, A., Tenenbaum, J.B.: Learning ovehypotheses with hierarchical Bayesian models. Dev. Science 10(3), 307-321 (2007)

23. Emmorey, K.: Space on hand: The exploitation of signing space to illustrate abstract thought. In: Gattis, M. (ed.) Spatial schemas and abstract thought, pp. 147-174. The MIT Press, Cambridge (2001) 